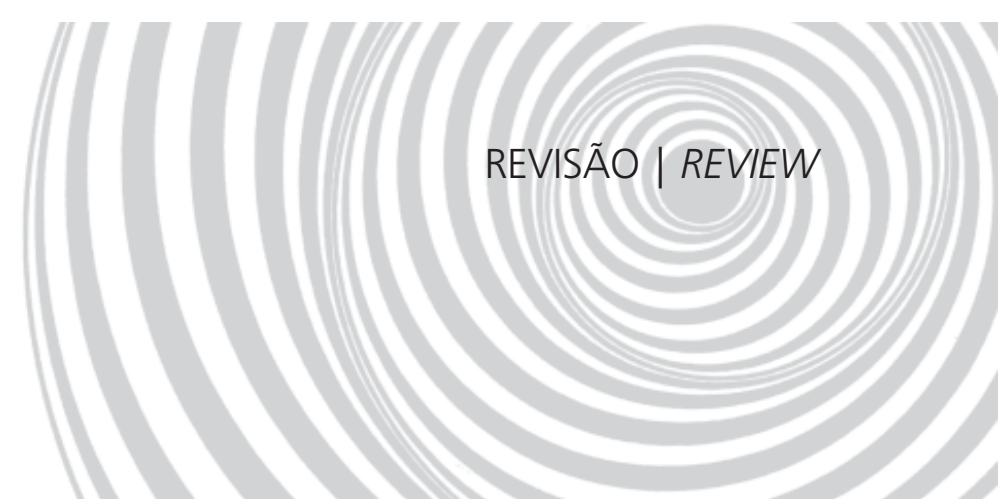

\title{
Caracterização dos estudos de avaliação do consumo alimentar de pré-escolares
}

\author{
Characterization of dietary intake assessment \\ studies in pre-school children
}

Renata Couto FALCÃO-GOMES ${ }^{1}$

Andréa Araciaba Soares COELHO ${ }^{1}$

Bethsáida de Abreu Soares SCHMITZ1,2

\section{RE S U M O}

Os estudos dietéticos fornecem elementos elucidativos que contribuem para a compreensão da dinâmica e determinação dos diversos agravos nutricionais que atingem a população infantil. As investigações do consumo alimentar de crianças pequenas utilizam os mesmos métodos de inquéritos empregados nas demais faixas etárias, diferenciando-se pela necessidade de um respondente, geralmente um dos pais ou o cuidador da criança. O objetivo do trabalho foi identificar os principais métodos de avaliação do consumo alimentar utilizados nos inquéritos com pré-escolares, assim como os itens e formas de análise mais comumente empregados. Verificou-se que os estudos apresentaram baixa comparabilidade, em função, entre outros fatores, do uso de diferentes formas de análise e metas de ingestão. O recordatório de $24 \mathrm{~h}$ foi o método mais utilizado, geralmente associado a um outro método de inquérito. Ainda são poucos os estudos que empregam as novas cotas dietéticas de referência para avaliação da adequação das dietas. No Brasil, assim como nos demais países em desenvolvimento, os estudos ainda priorizam as carências nutricionais. Apesar do aumento no número de estudos brasileiros nos últimos anos, as pesquisas existentes são de limitada comparabilidade, sendo essencial que se invista em uma estrutura (tabelas, softwares, seleção de indicadores) que possibilite um diagnóstico nutricional mais fidedigno e com potencial de comparação.

Termos de indexação: avaliação nutricional; consumo de alimentos; método de alimentação; pré-escolar.

\section{A B S T R A C T}

Dietary studies provide elucidative elements that contribute to the comprehension of both dynamics and determination of the several nutritional problems that afflict children of young age. Apart from the need of a

\footnotetext{
1 Programa de Pós-Graduação em Nutrição Humana, Departamento de Nutrição, Faculdade de Ciências da Saúde, Universidade de Brasília. Brasília, DF, Brasil.

2 Observatório de Políticas de Segurança Alimentar e Nutrição, Núcleo de Pesquisas em Políticas Públicas, Universidade de Brasília. Campus Universitário Darcy Ribeiro, Asa Norte, 70910-900, Brasília, DF, Brasil. Correspondência para/Correspondence to: B.A.S.SCHMITZ.E-mail: <bschmitz@unb.br>.
} 
respondent (generally one of the child's parent or a caregiver), dietary assessment of young children uses the same inquiry methods applied to people of other ages. The aim of this paper was to identify the most used inquiry methods applied in food consumption investigations of pre-school children, as well as the most common items and ways of analysis employed. It was observed that the studies presented a low comparability due to, among other factors, the use of different ways of analysis and ingestion goals. The 24-hours recall was the most applied method, frequently associated with another inquiry method. There are still few studies that use the new dietary reference intakes in dietary assessment. In Brazil, as in other developing countries, studies still give priority to nutritional deficiencies. Despite the growing number of Brazilian dietary studies in recent years, the existent researches are of limited level of comparability, being, therefore, crucial to invest in a structure (tables, software, indicator selection) that truly enables a more reliable and comparative nutritional diagnosis.

Indexing terms: nutrition assessment; food consumption; feeding methods; child, preschool.

\section{N T R O D U Ç Ã O}

Os estudos dietéticos têm entre seus objetivos, determinar os alimentos que constituem a dieta de um grupo ou indivíduo, fornecendo elementos elucidativos em relação à associação entre dieta e saúde, particularmente no que se refere à determinação dos diversos agravos nutricionais.

Para que ocorra uma correta avaliação do consumo alimentar, a escolha do método de investigação dietética deve estar pautada no objetivo da investigação (dieta total, alimentos ou grupos de alimentos, padrões e características da dieta, tipo de nutriente investigado) e nas características da população-alvo. Deve-se atentar para o fato de que não existe método de avaliação do consumo alimentar que seja considerado perfeito ${ }^{1}$. A capacidade do entrevistador em coletar os dados pode interferir, de forma contundente, na segurança das informações obtidas. O conhecimento teórico da metodologia escolhida, o treinamento prévio, a utilização adequada das técnicas de inquirir e o rigor científico são itens que devem ser cumpridos ${ }^{1,2}$.

A aceitabilidade do método pelo grupo avaliado está inversamente relacionada à sua precisão. Os métodos que apresentam um menor número de erros são os de maior custo, menor aceitabilidade, requerem mais tempo para execução, além de equipamentos mais sofisticados e recursos humanos altamente qualificados ${ }^{3}$.

Ao selecionar um método para avaliar o consumo alimentar, a duração da coleta deve ser condizente com o propósito da pesquisa. $\mathrm{Na}$ análise individual são necessárias mais observações do que se o foco for coletivo, devendo-se considerar a variabilidade intra e interindividual, o nutriente a ser investigado e o grau de exatidão desejado, como elementos que afetam o número de dias de observação ${ }^{4}$.

Os métodos de investigação dietética podem ser classificados em retrospectivos e prospectivos, sendo que a combinação de ambos também pode ser utilizada. O Recordatório de 24 horas (R24h), o Questionário de Freqüência Alimentar (QFA), que pode ser qualitativo, semi-quantitativo ou quantitativo, e a História Dietética são métodos retrospectivos. Para avaliação prospectiva podem ser utilizados a Pesagem Direta, os Registros Alimentar (estimado ou pesado), Telefônico, Fotográfico ou em Videoteipe, os Registros Eletrônicos, além da Análise de Duplicatas de Porções e da Observação direta por registro de vídeo ou Observação Direta por observadores treinados ${ }^{1,2,4}$. O conhecimento da descrição básica, das vantagens e desvantagens de cada método e dos cuidados na coleta de informações auxilia na determinação de sua utilização, uma vez que cada método tem características próprias, já tendo estas sido descritas amplamente na literatura ${ }^{3-5}$.

No caso da população infantil, deve-se ter cuidado especial na escolha do(s) método(s), pois este grupo etário, em geral, necessita de um respondente, principalmente os pré-escolares. Em relação aos escolares, verifica-se que possuem vocabulário limitado, com pouca capacidade de 
descrever os alimentos e de quantificar porções. As crianças também têm sua atenção desviada rapidamente para outros assuntos de interesse próprio, o que pode dificultar a entrevista.

A qualidade da resposta melhora com a idade. Entre os 7 e 8 anos de idade há um rápido aumento na capacidade da criança de responder de forma confiável, e por volta dos 10 a 12 anos, elas já estão aptas a responder com precisão ${ }^{3}$.

Dessa forma, estudos epidemiológicos sobre dietas de crianças mais novas apresentam dificuldades adicionais em relação aos de adultos, embora possam utilizar os mesmos métodos de inquérito. Para a obtenção de dados confiáveis torna-se imprescindível, além da seleção do método adequado, a utilização de instrumentos especialmente desenhados para a população infantil. É necessário considerar a grande variação diária do consumo, que ocorre em algumas faixas etárias, a rápida mudança dos hábitos alimentares e a incapacidade cognitiva da criança de retratar fidedignamente sua alimentação, tornando-se imprescindível a participação dos pais, responsáveis ou cuidadores da criança. A qualidade das informações fornecidas por estes também pode ser influenciada por fatores, como motivação, memória e nível de escolaridade ${ }^{6,7}$.

Apesar das dificuldades mencionadas, a avaliação do padrão alimentar dos pré-escolares é de primordial importância face à relevância da composição da dieta infantil para a manutenção de um estado nutricional adequado. Doenças carenciais, como anemia ferropriva, deficiência de vitamina A e desnutrição, estão epidemiologicamente associadas a essa faixa etária. Por sua vez, hábitos alimentares errôneos contribuem para o incremento do sobrepeso e obesidade em crianças mais precocemente. Além de acarretar o comprometimento do crescimento e desenvolvimento na infância, o consumo inadequado de alimentos pode antecipar doenças da fase adulta, como hipertensão e diabetes tipo 2, ou aumentar o risco para o posterior aparecimento de doenças cardíacas, osteoporose e câncer ${ }^{6}$.
A identificação do perfil de consumo alimentar constitui, portanto, uma etapa fundamental para o estabelecimento de estratégias que objetivem reverter o atual quadro epidemiológico nutricional, visando à promoção da saúde e da alimentação saudável.

Este trabalho tem o intuito de identificar os principais métodos de avaliação de consumo alimentar utilizados nos inquéritos alimentares em pré-escolares, assim como os itens e formas de análise mais comumente usadas.

\section{MÉTODOS}

A metodologia utilizada foi a pesquisa em base de dados Medline (Literatura Científica Internacional) e Lilacs (Literatura Latino-Americana e do Caribe em Ciências da Saúde) e publicações específicas da área de Nutrição, considerando o período de 1997 até julho de 2005. Foram incluídos os artigos que tiveram como objetivo a análise do consumo alimentar retrospectivo e prospectivo em pré-escolares, aqui considerados como crianças menores de 7 anos, e excluídos os estudos de validação e de intervenção. Foram encontrados e analisados 33 estudos que se enquadravam no propósito da revisão, e cujas principais características encontram-se em Anexo.

Foram identificadas como variáveis de observação para essa caracterização, os autores, ano de publicação, país de realização da pesquisa, população investigada (amostra e faixa etária), método utilizado para avaliar o consumo alimentar, item(s) de consumo analisado(s), nutriente, alimento, grupo de alimento, e forma de análise.

Após a caracterização inicial, os trabalhos foram classificados segundo o principal tema discutido nos artigos e o local de origem, se oriundos de países desenvolvidos ou em desenvolvimento.

Para que se faça a análise dos diversos estudos, deve-se ter como pressuposto a comparabilidade. Por outro lado, a comparabilidade dos inquéritos dietéticos depende, entre outros fatores, 
da população estudada, do tipo de método utilizado, do tipo de categorização dos alimentos e da base de dados utilizada para a composição dos alimentos ${ }^{8}$.

\section{População}

O número de crianças pesquisadas ${ }^{9}, 10 \mathrm{em}$ cada estudo variou de 51 a 8 044. Essa ampla variação se explica porque alguns estudos foram realizados em instituições (creches, centros de saúde) e outros eram pesquisas de âmbito nacional.

O número de indivíduos pesquisados tem repercussões metodológicas. A variação relativa ao indivíduo é, geralmente, muito maior do que a variação verificada entre os indivíduos, e se altera dependendo da população estudada e do método utilizado. Para que se tenham dados mais confiáveis, pode-se utilizar um maior número de dias. Por sua vez, a variação interindividual varia para cada nutriente e aumenta com a heterogeneidade da população que está sendo estudada. Na presença de grande variação interindividual, faz-se necessário o aumento do número amostral².

Os estudos foram realizados em pré-escolares de diferentes faixas etárias, sendo que 15 $(45,4 \%)$ avaliaram dietas de crianças a partir de 24 meses de idade. Alguns utilizaram uma idade específica para o estudo, sendo a mais comum a de 18 meses. A existência de várias faixas etárias compromete a comparabilidade, uma vez que a alimentação das crianças não se comporta de maneira homogênea na fase pré-escolar. A alimentação das crianças mais novas muda muito nos primeiros dois anos de vida. Neste período há também o problema do registro adequado do consumo de leite humano, sendo este um dos motivos de alguns inquéritos excluírem as crianças que estão em aleitamento materno.

A comparabilidade entre os estudos que analisaram o consumo do leite materno ficou prejudicada, em função do uso de diferentes valores para média de ingestão, utilizados para as diferentes faixas etárias ${ }^{11-14}$. Dez estudos que incluíram crianças menores de dois anos em sua amostra excluíram aquelas em aleitamento materno, ou não consideraram, ou ainda simplesmente não relataram a forma como analisaram o consumo de leite materno $0^{9,10,15-22}$. É conhecida a relevância do aleitamento materno para o estado nutricional de menores de dois anos, principalmente em países em desenvolvimento. A ausência da análise desse consumo pode ter influenciado os resultados.

Ramakrishnan et al. ${ }^{11}$, analisando o consumo de vitamina A em crianças indianas de 12 a 47 meses, observaram que o leite materno era uma importante fonte de retinol para a dieta dessas crianças. De fato, três estudos que investigaram o consumo de vitamina A recomendaram, em suas conclusões, o estímulo ao aleitamento materno como forma de prevenir a deficiência desse nutriente ${ }^{10,11,18}$.

Livingstone \& Robson ${ }^{23}$, em um artigo de revisão sobre investigações de consumo alimentar em crianças, verificaram que, após os dois primeiros anos, a alimentação mantém-se razoavelmente constante até os cinco anos de vida, quando volta a ter mais variações de consumo. Aparentemente, nessa fase a criança ajusta sua ingestão energética ao longo do dia, tornando-a relativamente constante. Em crianças maiores de cinco anos a relação entre a variabilidade intraindividual e a variação entre os indivíduos é, aproximadamente, o dobro da que é observada em adultos, gerando repercussões sobre o tipo de método a ser utilizado e sobre o número de dias a ser pesquisado. Para as crianças mais novas (menores de quatro anos), devido à variação intraindividual ser relativamente mais baixa, o registro alimentar de sete dias é considerado adequado para relatar o consumo em relação a energia e à maioria dos nutrientes.

\section{Métodos utilizados pelos estudos}

O R24h, aplicado por 1 dia, em intervalos regulares, ou em 2 ou 3 dias, consecutivos ou 
não, foi o método mais utilizado $(43,7 \%)$, geralmente associado a outro. Quando bem conduzido, o R24h fornece informações sobre a ingestão média das populações. Não é um método acurado para medir ingestões individuais quando aplicado uma única vez, em função da variabilidade intraindividual da dieta. Para conhecer a ingestão individual habitual de energia e macronutrientes é necessário que seja repetido de 3 a 4 vezes, em dias não consecutivos ${ }^{4,24}$. Para avaliação de dietas de grupos, aparentemente, a distribuição do consumo usual em percentis é semelhante para 2 ou 7 dias, sugerindo que mais de duas medições por indivíduo seja desnecessário ${ }^{25}$.

O registro alimentar, geralmente de três dias, e utilizando a pesagem de alimentos, e o QFA semiquantitativo também foram muito utilizados. O QFA foi o instrumento de escolha para avaliação de vitamina A. A qualidade da informação obtida por meio de um QFA é diretamente proporcional à seleção adequada dos alimentos incluídos e à estimativa das porções. Quando aplicado em crianças, o QFA deve listar os alimentos que habitualmente são consumidos por este grupo inclusive lanches feitos fora de casa e guloseimas, como salgadinhos, biscoitos, balas, doces e refrigerantes ${ }^{6,7}$. O QFA recomendado pelo International Vitamin A Consultative Group (IVACG) foi utilizado em alguns estudos com vitamina $A^{18,26-28}$, sendo adaptado para incluir alimentos regionais.

Humphrey et al. ${ }^{29}$ compararam o uso do QFA IVACG e um R24h em pré-escolares com deficiência de vitamina A. Eles elaboraram um R24h com porções pré-determinadas de alimentos fontes de vitamina $A$, obtidas junto à população local. Esse recordatório se associou significantemente com o retinol sérico, enquanto o QFA IVACG apresentou um consumo que não se correlacionou com a concentração sérica daquele composto, sugerindo que tal instrumento superestimou o consumo. Os autores consideraram que a memória recente possibilita dados mais precisos, principalmente quando se lida com populações pouco instruídas.
Oito estudos desta revisão utilizaram o registro alimentar como único método de consumo, refletindo a confiabilidade dos pesquisadores no método, por propiciar um relato preciso da dieta de uma pessoa por um período específico ${ }^{9}, 12,17,19,20,27,28,30$. Em investigações com pré-escolares, os alimentos são registrados pelos pais ou cuidadores da criança. O método demanda um bom nível educacional e cooperação ${ }^{7}$.

A pesagem direta foi utilizada somente em creches. É um método que necessita de mais tempo para execução, tem maior custo e gera transtornos à rotina. Requer equipamentos precisos, questionários adequados e pessoal treinado. A precisão do método depende também do número de dias de observação. Para obter o consumo alimentar médio de um grupo de indivíduos, deve ser aplicado durante três dias não consecutivos da semana',31.

A aplicação desse método é facilitada quando ele é empregado em populações institucionalizadas, quando a pesagem direta pode ser utilizada para coletar informações que permitam estimar níveis de consumo médio do grupo e sua distribuição, ou obter valores médios do consumo individual ${ }^{32}$. Estudo realizado em creches de São Paulo recomenda que seja evitado o método de pesagem direta total, devido à baixa concordância encontrada entre este método e a pesagem direta individual. Na pesagem total, a quantidade média de alimentos oferecida às crianças foi obtida indiretamente a partir do total de alimentos preparados, descontando-se a sobra limpa e a quantidade consumida pelos adultos. Na individual, em todas as refeições, foi pesada a quantidade média de cada alimento oferecido à criança, descontando-se os restos ${ }^{31}$.

Os métodos menos utilizados foram a análise química $(12,1 \%)$, a história dietética $(6,0 \%)$ e a observação direta $(3,0 \%)$.

\section{Tipo de estudo e forma de análise}

Os estudos foram predominantemente transversais (75,7\%), o que se justifica por serem 
mais rápidos, de menor custo e melhor operacionalidade.

A forma de análise dos resultados variou segundo o objetivo dos estudos. De maneira geral, obteve-se a média ou mediana diária de ingestão de nutrientes, ou ainda o percentual de nutrientes e energia em relação ao valor energético total (VET). Os QFA foram analisados segundo o consumo mensal ou semanal de grupos de alimentos. Foram estabelecidas diferentes categorizações de alimentos nos diversos instrumentos que utilizaram análise por grupos de alimentos, mesmo quando analisavam o mesmo nutriente ou tipo de alimento (ex. alimentos líquidos), o que dificulta a comparabilidade dos estudos. Além disso, os alimentos foram convertidos em nutrientes segundo diferentes tabelas de composição de alimentos e diferentes bases de dados, fato observado, inclusive, em estudos de um mesmo País, como, por exemplo, o Brasil.

A adequação dietética também foi feita baseada em diversas metas de ingestão. As mais utilizadas foram a Recommended Dietary Allowance (RDA) americana, em estudos nos Estados Unidos e em países em desenvolvimento, e as Recommended Nutrient Intake (RNI) britânicas. Outros estudos utilizaram recomendações próprias para suas populações, como a Ingestão Recomendada (RI) espanhola e a Recommended Nutrition Intake (RNI) canadense. Isso dificulta a comparabilidade porque, o que é considerado adequado em um estudo, com base em suas recomendações próprias, pode não ser adequado segundo as recomendações de outro País. Por exemplo, a RDA para ferro é de $10 \mathrm{mg}$ para crianças maiores de 6 meses, enquanto que a RNI canadense para ferro é de $7 \mathrm{mg}$ para crianças de 5 a 11 meses e de $6 \mathrm{mg}$ para maiores de 12 meses. A diferença nas recomendações deve-se ao fato de que a RDA baseou-se em crianças com aleitamento artificial, enquanto que a RNI canadense baseou-se em crianças em aleitamento materno e considerou a biodisponibilidade do leite materno ${ }^{33}$.

Em relação à adequação, observou-se que ela se deu sob a forma percentual ou pelo estabelecimento de níveis ideais de consumo de nutrientes ou tipo de alimento segundo diversas variáveis (idade, quartil de ingestão lipídica, faixa de renda), indicando diferentes formas de análise.

A adequação percentual dos nutrientes em relação à RDA não é a forma mais recomendada atualmente para avaliar adequação da dieta de grupos, visto que esta abordagem pode resultar em superestimação. Como a variância da ingestão na população, geralmente, excede a variância das necessidades para a maioria dos nutrientes, a média da ingestão habitual do grupo necessita exceder a RDA, às vezes com uma margem considerável, para que haja uma baixa prevalência de ingestão inadequada ${ }^{34,35}$. Para avaliação de dietas de grupos e indivíduos recomenda-se o uso das Dietary Reference Intakes (DRI) como referência, sendo que a análise da necessidade média estimada Estimated Average Requirement (EAR) é a mais indicada para expressar a prevalência de inadequação da ingestão dentro de um grupo. Para estimar o percentual da população em risco potencial de consumo excessivo de um nutriente é recomendado utilizar o limite superior tolerável de ingestão Tolerable Upper Intake Level (UL).

Nesta revisão, chama a atenção o fato de apenas dois estudos terem utilizado essa metodologia de análise. Arsenault \& Brown encontraram, em uma população de crianças americanas menores de 6 anos, um consumo de zinco acima da UL. O percentual de crianças com o consumo excedente decresceu com a idade, indo de $92,0 \%$ aos 6 meses a 3,0\% aos 4-5 anos. Esses autores utilizaram dois R24h para obter a variabilidade intrapessoal e entre indivíduos ${ }^{22}$.

No Brasil, estudo realizado com crianças que freqüentam creches mostrou que, quando comparados aos valores de referência das novas DRI, com exceção da proteína, houve inadequação no consumo de vitamina $C$, ferro, vitamina A e cálcio relativamente à necessidade média estimada (EAR). Além disso, verificou-se que 75,7\% das crianças apresentaram inadequação energética em relação à necessidade energética estimada $(E E R)^{36}$. No entanto, não foi realizado, 
no citado estudo, o ajuste da distribuição da ingestão usual dos nutrientes no grupo, procedimento estatístico preconizado pelo Institute of Medicine, para remover parcialmente a variabilidade intraindividual.

\section{Local de realização e objeto de estudo}

Observa-se, nos dados da Tabela 1 que, nos países desenvolvidos, os estudos recentes enfocam, principalmente, os alimentos e nutrientes envolvidos na determinação de doenças crônicas não transmissíveis, pelo excesso de ingestão (lipídeos, ingestão de alimentos líquidos) ou pela deficiência de consumo (fibras, frutas e vegetais). A presença de apenas quatro artigos naqueles países para conhecer o padrão geral de ingestão alimentar (na população ou em coletividades, como nas creches), justifica-se pela realização contínua de pesquisas nacionais, ao contrário dos países em desenvolvimento que ainda têm necessidade de traçar o perfil de consumo básico de suas populações. Nos Estados Unidos, desde 1940 são realizados estudos populacionais (Longitudinal Studies of Child Health and Development) sendo que a partir dos anos 60, o governo americano iniciou uma série de pesquisas nacionais para caracterizar o estado nutricional dos americanos, tendo como base o consumo alimentar (National Health and Nutrition
Examination Surveys-I, II e III, Nationwide Food Consumption Surveys- I e II $)^{7}$. Na Europa, vários governos também investiram em estudos de consumo a partir dos anos $70 \mathrm{e}$, atualmente, os esforços são para uniformizá-los, uma vez que os grupos populacionais avaliados, as faixas etárias e os métodos dietéticos empregados diferem grandemente entre os países ${ }^{8}$. Devido à alta complexidade e ao elevado custo, poucas nações em desenvolvimento realizam como rotina inquéritos nacionais de consumo. Dos estudos que avaliaram um grande número de crianças ( $n>1000)$, apenas um foi realizado em país não desenvolvido ${ }^{26}$.

Os estudos existentes nesses países enfatizaram, além da determinação do padrão de consumo básico dessa população, as doenças carenciais, investigando nutrientes envolvidos ou relacionando a ingestão desses com o estado nutricional. Vale ressaltar que o ferro, como elemento limitante, foi um nutriente investigado por diversos países, independentemente do nível de desenvolvimento.

\section{Caracterização e identificação de viéses}

Erros sistemáticos são intrínsecos aos métodos de avaliação de consumo alimentar e devem ser considerados em todas as etapas dos inquéritos dietéticos, visando evitar conclusões

Tabela 1. Caracterização dos artigos segundo tema principal de estudo e país de realização da pesquisa, 1997-2005.

\begin{tabular}{|c|c|c|c|c|}
\hline \multirow{2}{*}{ Tema } & \multicolumn{2}{|r|}{ País desenvolvido } & \multicolumn{2}{|c|}{ País em desenvolvimento } \\
\hline & $\mathrm{n}$ & País & $n$ & País \\
\hline Ingestão de nutrientes (geral) & 2 & Estados Unidos, Hong Kong & 3 & Brasil, México, Gana \\
\hline Consumo em creches & 2 & Estados Unidos & 5 & Brasil \\
\hline Ingestão de alimentos líquidos & 2 & Estados Unidos, Inglaterra & & \\
\hline Ingestão lipídica & 4 & Espanha, Hong Kong, Inglaterra & 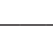 & $\longrightarrow$ \\
\hline Ferro/anemia & 3 & Canadá, Inglaterra, Nova Zelândia & 3 & Brasil, Irã \\
\hline Vitamina $\mathrm{A} /$ hipovitaminose $\mathrm{A}$ & 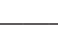 & $\longrightarrow$ & 5 & Brasil, Índia \\
\hline Alimentação complementar & 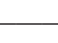 & $\longrightarrow$ & 1 & Malásia \\
\hline Frutas e vegetais & 1 & Estados Unidos & . & \\
\hline Fibras & 1 & Bélgica & 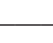 & $\longrightarrow$ \\
\hline Zinco & 1 & Estados Unidos & 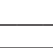 & \\
\hline Total & 16 & & 17 & \\
\hline
\end{tabular}


equivocadas. O planejamento minucioso contribui para verificar a existência de possíveis fontes de variação e elaborar estratégias para minimizá-las.

A associação de métodos contribui para a exatidão. Como não existe um método de avaliação do consumo alimentar que seja considerado perfeito, isento de desvantagens e viéses, é notório o uso de combinação entre distintos tipos de inquéritos. Isso também tem sido observado em estudos voltados para o consumo alimentar de crianças e foi uma estratégia utilizada por 13 $(39,4 \%)$ dos estudos analisados. Como exemplo, verificou-se que pesquisas realizadas na China ${ }^{15,16}$ utilizaram a História Dietética, o Questionário de
Freqüência Alimentar e o Recordatório de 24 horas associados, visando a conferir exatidão ao consumo mensurado. Em Gana, Takyi ${ }^{37}$ utilizou a combinação entre QFA e R24 horas, sendo que o recordatório foi aplicado em dois dias da semana não consecutivos e em um dia no final de semana. Essa alternativa de utilização do recordatório objetiva avaliar tanto a ingestão usual quanto a atípica, mensurando a média de consumo alimentar.

O Quadro 1 expõe os viéses que podem permear os vários métodos de avaliação do consumo alimentar.

Quadro 1. Viéses encontrados em métodos de avaliação de consumo alimentar.

\begin{tabular}{|c|c|c|}
\hline Viés & Fonte & Método \\
\hline Seleção & Erro de amostragem & Todos \\
\hline \multirow[t]{18}{*}{ Aferição } & Erro de resposta & \\
\hline & Sub ou superestimação de consumo & R24h, QFA, história dietética, registro alimentar \\
\hline & Estimativa de pesos e porções & $\begin{array}{l}\text { R24h, história dietética, QFA e registro alimentar } \\
\text { estimado }\end{array}$ \\
\hline & Estimativa de freqüência de consumo de alimentos & História dietética, QFA \\
\hline & Variação de dia para dia & $\begin{array}{l}\text { Análise em duplicata, registro alimentar, R24h, } \\
\text { pesagem direta }\end{array}$ \\
\hline & Erro do entrevistador ou pesquisador & \\
\hline & Registro incorreto das respostas & Todos \\
\hline & Descrição incompleta dos alimentos & R24h, história dietética \\
\hline & Erros de codificação & Todos, exceto, análise em duplicata \\
\hline & Erros na conversão de medidas caseiras em gramas & R24h, história dietética, registro alimentar estimado \\
\hline & Erros na conversão de alimentos em nutrientes & \\
\hline & Análise direta & Análise em duplicata \\
\hline & Tabelas de composição de alimentos & Todos, menos análise em duplicata \\
\hline & $\begin{array}{l}\text { Valores dos bancos de dados de nutrientes usados para } \\
\text { grupos de alimentos }\end{array}$ & QFA \\
\hline & Erros de computação & Todos \\
\hline & Erros de instrumento & \\
\hline & Balanças descalibradas & Todos que exigem pesagem \\
\hline & Medidas caseiras não padronizadas & R24h, história dietética, registro alimentar estimado \\
\hline Memória & Falha de memória & R24h, QFA, história dietética \\
\hline Adesão & Colaboração voluntária & Todos, exceto análise em duplicata \\
\hline
\end{tabular}

Adaptado de: Kanimura et al. ${ }^{1}$ e Dwyer ${ }^{2}$. 
Com vistas a diminuir erros de amostragem, evitando que a população selecionada para os estudos gerasse diferenças entre as ingestões reais e as medidas, não representando o grupo populacional pesquisado, 90,6\% dos autores optaram pela aleatoriedade, selecionando indivíduos que eram representativos. Apenas três estudos escolheram as crianças participantes utilizando como único critério a aceitação dos pais $^{9,12,38}$. Essa forma de seleção aglutina dois viéses, o de seleção e o de cooperação, podendo produzir erros potenciais ao estudo, inclusive, comprometendo sua capacidade de generalização.

Uma das dificuldades na aplicação dos inquéritos dietéticos é, justamente, a cooperação dos avaliados, pois a participação nos inquéritos demanda tempo e paciência. Nos pré-escolares, a adesão dos pais ou cuidadores é imprescindível, uma vez que eles são os respondentes, em virtude da capacidade limitada de resposta das crianças menores de sete anos.

A cooperação pode ser medida por meio da taxa de adesão (response rate). A taxa de adesão, avaliada em $24,2 \%$ dos artigos estudados, variou de 67 a $85 \%$, sendo que o valor mais alto correspondeu ao estudo no qual se verificou viés de seleção, com indicação do diretor da creche para os respondentes mais cooperativos. Todos os estudos que forneceram a taxa de adesão utilizaram o registro alimentar $9,10,17,19,20,30,39,40$. Uma questão que preocupa o pesquisador é o quanto seus resultados podem ser afetados pela adesão, na medida em que os indivíduos que não respondem poderiam ser, no caso de pré-escolares, por exemplo, os pais das crianças mais afetadas por distúrbios nutricionais ou que tivessem uma alimentação mais desequilibrada. O ideal seria traçar um perfil dos que se recusam a participar da pesquisa e tentar conhecer os motivos para a falta de cooperação. Dennison et al. ${ }^{39}$ tiveram oportunidade de analisar algumas características dos respondentes e não respondentes, em um estudo sobre consumo de frutas e vegetais que utilizou registro alimentar de sete dias conse- cutivos. Os autores verificaram que, normalmente, quem respondia era a mãe. Em relação às que não cooperaram, as mães respondentes possuíam melhor nível educacional, tinham menor probabilidade de fumar e de participar de programas de suplementação nutricional para mulheres e crianças $(p<0,05)$. Não foram encontradas diferenças nas medidas antropométricas entre as crianças das mães que cooperaram ou não.

Os viéses que mais contribuem para erros nos inquéritos de consumo são os de aferição, podendo acarretar sub ou superestimação do consumo. A dificuldade de estimar com exatidão a dieta reside em aspectos relacionados à variação real dos alimentos consumidos pelos indivíduos, devido à diversificação, à heterogeneidade e às flutuações da alimentação do dia-a-dia, bem como às variações relativas ao próprio processo de mensuração ${ }^{34}$.

Beaton et al. ${ }^{41}$, ao revisarem os resultados de um estudo de validação de um R24h, verificaram uma subestimação superior a $25 \%$. Além disso, constataram que, nas pesquisas populacionais americanas, a subestimação era maior nos indivíduos com índice de massa corporal (IMC) mais elevado. Em crianças pequenas, a influência da adiposidade dos pais na exatidão das respostas é inconsistente, parecendo estar mais relacionada a crianças obesas que têm, pelo menos, um dos pais com obesidade ${ }^{23}$.

O fato de os inquéritos dietéticos em pré-escolares se basearem em relato ou registro feito pelos pais, pode contribuir para erros, na medida em que os mesmos não são confiáveis para relatar o consumo da criança fora de casa. Mães de pré-escolares que se ausentam de casa por mais de quatro horas por dia são menos capazes de relatar o consumo alimentar de suas crianças. Para o R24h, o relato mais fidedigno parece ser obtido quando os dois responsáveis pela criança participam, sendo considerada limitante a participação de um cuidador como respondente ${ }^{23}$.

Um aspecto que contribui para a impressão do depoimento é o desejo de ajuste social, que consiste na tendência de o indivíduo fornecer a 
resposta mais aceitável e desejável socialmente, independentemente de ser verdadeira ou não, agregando ao relato condicionantes morais, emocionais e sociais ${ }^{42}$.

Esses aspectos são particularmente importantes em relação à alimentação infantil, principalmente em populações com níveis consideráveis de insegurança alimentar, nas quais a mãe (ou responsável) pode não relatar o consumo de alimentos que crê serem prejudiciais à criança, mas que a mesma consumiu por serem os únicos disponíveis no domicílio, ou até fantasiar refeições completas para ocultar a ausência do consumo, por vergonha de relatar a fome.

Nesse contexto, torna-se necessária a adoção de métodos de análise capazes de apreender os diversos fatores determinantes do consumo e de sua evolução, de natureza econômica, social, cultural, política e nutricional, bem como a interação entre eles ${ }^{43}$.

Nos estudos analisados, as estratégias mais utilizadas para minimizar os viéses de aferição foram: treinamento dos entrevistadores, motivação, treinamento e esclarecimento aos pais sobre a pesquisa e o uso correto da balança e de medidas caseiras, utilização de fotos ou desenhos de utensílios e porções, distribuição de balanças, coleta de dados em dias não consecutivos, incluindo pelo menos um final de semana, visitas domiciliares para confirmar informações e reforçar orientações, além da revisão dos questionários por nutricionistas para avaliação de consistência. Outras estratégias observadas para o maior controle de qualidade das informações são descritas a seguir.

Takyi ${ }^{37}$ limitou em 10 o número de questionários a serem aplicados diariamente pelos entrevistadores, como forma de garantir a qualidade das informações. Leung et al. ${ }^{15}$, além de checarem a história dietética com a aplicação de um QFA e um R24h, realizaram visitas para observar discrepâncias e analisar as despesas dos domicílios com alimentos, como forma indireta de checar o consumo. No Canadá, Goodwin et al. ${ }^{33}$, ao aplicarem, num estudo de coorte, uma série de R24h, optaram por realizar um segundo R24h em 25\% das crianças, como forma de ajustar a variabilidade intraindividual, após cada aplicação dos inquéritos previstos.

Três estudos utilizaram métodos para verificar a acurácia dos instrumentos de coleta em estimar a ingestão real $30,40,44$, sendo que Muñoz et al. ${ }^{40}$ encontraram uma freqüência de 10\% de subestimação do consumo em crianças americanas menores de 12 anos.

Alguns estudos que utilizaram o registro alimentar aplicaram estratégias visando a diminuir o sub-registro. Os respondentes eram incentivados a fazer o registro logo após as refeições para evitar omissão, e a guardar latas, embalagens e rótulos dos alimentos consumidos. Para facilitar o registro, os participantes recebiam um diário alimentar pré estruturado, que era checado logo após a entrega, por entrevistadores treinados $12,20,45$.

\section{CONSIDERAÇÕES FINAIS}

As informações dietéticas fornecem subsídios para a identificação de comportamentos e hábitos alimentares. Entretanto, os dados devem ser interpretados cautelosamente, servindo muito mais para indicar um risco nutricional, relacionado à carência ou ao excesso alimentar, do que para diagnosticá-lo, pois o consumo é um indicador indireto do estado nutricional. Além disso, as necessidades individuais de nutrientes podem se diferenciar substancialmente das recomendações diárias ${ }^{46}$

Apesar das dificuldades metodológicas da obtenção de dados de consumo, em relação a outros indicadores nutricionais, a elevada prevalência de doenças associadas à dieta, presente tanto em países desenvolvidos como nos em desenvolvimento, justifica o investimento nesse tipo de pesquisa.

Entre os artigos investigados ainda foi incipiente o uso dos novos valores de referência, as DRI, talvez porque só recentemente, em 2002, é que o Institute of Medicine publicou o 
documento relativo à análise de energia, macronutrientes, fibras, colesterol, ácidos graxos e aminoácidos.

Fica evidente o aumento do interesse dos pesquisadores brasileiros na investigação do consumo alimentar de pré-escolares. Dos 11 artigos brasileiros, 9 foram publicados a partir de 2000 . Salienta-se que a faixa etária estudada é de grande vulnerabilidade biológica, estando sujeita aos diversos agravos nutricionais. Mais recentemente, se observa uma crescente preocupação com o incremento da obesidade infantil no País. Apesar dessa realidade epidemiológica, os estudos brasileiros ainda priorizam as doenças carenciais. Além disso, mostraram ser de limitada comparabilidade, por utilizarem diferentes indicadores, tabelas e programas de análise de dados, além da desagregação dos mesmos. Ressalte-se que os estudos com vitamina A apresentaram uma melhor comparabilidade, por se basearem em um mesmo protocolo internacional.

São necessários investimento em pesquisa, visando ao desenvolvimento e aperfeiçoamento de softwares adequados para análise de dados de consumo alimentar, e a utilização pelos profissionais de tabelas de alimentos mais completas, com dados mais confiáveis e adequados ao País. Nesse sentido, recentemente foi concluído o projeto Tabela de Composição de Alimentos (TACO).

Face à dificuldade de implementação de grandes pesquisas nacionais, a alternativa de estudos multicêntricos, aliada a investigações pontuais, mas comparáveis, poderia responder a muitos questionamentos sobre a dieta e sua repercussão sobre a saúde infantil.

\section{REFERÊ N CIAS}

1. Kanimura MA, Bakmann A, Sampaio LR, Cuppari L. Avaliação Nutricional. In: Cuppari L. Nutrição clínica no adulto. São Paulo: Manole; 2002. p.91-4.

2. Dwyer J. Avaliação do consumo alimentar. In: Shils ME, Olson JÁ, Shike M. Tratado de nutrição moderna na saúde e na doença. Barueri: Manole; 2003. p.1001-26.

3. Pereira AML, Fisberg RM, Brasil ALD, Abreu VJS, Pacheco MEMS, Vasconcelos MIL. Métodos para avaliação do consumo alimentar em crianças e adolescentes. Rev Paul Pediatria. 1997; 15:210-4.

4. World Health Organization. Preparation and use of food-based dietary guidelines. Geneva; 1998. Technical Report Series, 880.

5. Cintra IP, Vonder Heyde MED, Schmitz BA, Franceschini SCC, Taddei JAAC, Sigulem DM. Métodos de Inquéritos Dietéticos. Cad Nutr. 1997; 13:11-23

6. Philippi ST. Transição no consumo alimentar de crianças de 0 a 59 meses na cidade de São Paulo [tese]. São Paulo: Universidade de São Paulo; 2001.

7. Rockett HRM, Colditz GA. Assessing diets of children and adolescents. Am J Clin Nutr. 1997; 65(4 Suppl):1116S-22S.

8. Verger PH, Ireland J, Moller A, Abravicius JA, DeHenauws S, Naska A. Improvement of comparability of dietary intake assessment using currently available individual food consumption surveys. Eur J Clin Nutr. 2002; 56(Suppl 2):S18-24.

9. Briley ME, Jastrow S, Vickers J, Roberts-Gray C. Dietary intake at child-care centers and away: are parents and care providers working as partners or at cross-purposes? J Am Diet Assoc. 1999; 99(8):950-4.

10. Rampersaud GC, Bailey LB, Kauwell GPA. National survey beverage consumption data for children and adolescents indicate the need to encourage a shift toward more nutritive beverages. J Am Diet Assoc. 2003; 103(1):97-100

11. Ramakrishnan U, Martorell K, Latham MC, Abel R. Dietary vitamin A intakes of preschool-age children in South India. J Nutr. 1999; 129(11):2021-7.

12. Heath ALM, Tuttle CR, Simos MSL, Cleghorn CL, Parnell WR. Longitudinal study of diet and iron deficiency anaemia in infants during the first two years of life. Asia Pacific J Clin Nutr. 2002; 11(40):251-7.

13. Hotz C, Gibson RS. Complementary feeding practices and dietary intakes from complementary foods amongst weanlings in rural Malawi. Eur J Clin Nutr. 2001; 55(10):841-9.

14. Soares NT, Guimarães ARP, Sampaio HAC, Almeida PC, Coelho RR. Padrão alimentar de lactentes residentes em áreas periféricas de Fortaleza. Rev Nutr. 2000; 13(3):167-76.

15. Leung SS, Lee WT, Lui SS, Ng MY, Peng XH, Luo HY, et al. Fat intake in Hong Kong Chinese children. Am J Clin Nutr. 2000; 72 (5 Suppl):1373S-8S. 
16. Leung SS, Chan SM, Lui S, Lee WT, Davies DP. Growth and nutrition of Hong Kong children aged 0-7 years. J Paediatr Child Health. 2000; 36(1): 56-65.

17. Northstone K, Rogers I, Emmet P. Drinks consumed by 18-month-old children: are current recommendations being followed ? Eur J Clin Nutr. 2002; 56(3):236-44

18. Assis AMO, Prado MS, Freitas MCS, Cruz MM. Deficiência de vitamina A e desnutrição energético protéica em crianças de localidades do semi-árido baiano. Rev Nutr. 1997; 10(1):70-8.

19. Rogers I, Emmet P. Fat content of the diet among pre-school children in Britain; relationship with food and nutrient intakes. Eur J Clin Nutr. 2002; 56(3): 252-63.

20. Cowin I, Emond A, Emmet P. Alspac study team. Association between composition of the diet and haemoglobin and ferritin levels in 18-month-old children. Eur J Clin Nutr. 2001; 55(4):278-86.

21. Lacerda E, Cunha AJ. Anemia ferropriva no segundo ano de vida no Rio de Janeiro, Brasil. Rev Panam Salud Publica. 2001; 9(5):294-301.

22. Arsenault JE, Brown KH. Zinc intake of US preschool children exceeds new dietary reference intakes. Am J Clin Nutr. 2003; 78(5):1011-7.

23. Livingstone MBE, Robson PJ. Measurement of dietary intake in children. Proc Nutr Soc. 2000; 59(2):279-93.

24. Beaton GH, Milner J, McGuire V, Feather TE, Little $J A$. Source of variance in 24-hour dietary recall data: implications for nutrition study design and interpretation. Carbohydrate sources, vitamins, and minerals. Am J Clin Nutr. 1983; 37(6):986-95

25. Hoffmann K, Boeing $H$, Dufour A, Volatier JL, Telman J, Virtanem $M$, et al. Estimating the distribution of usual dietary intake by short-term measurements. Eur J Clin Nutr. 2002; 56 (Suppl 2):S53-62.

26. Khandait DW, Vasudeo ND, Zodpeys P, Ambadekar NN, Koram MR. Vitamin A intake and xerophthalmia among Indian children. Public Health. 1999; 113(2):69-72.

27. Araújo KC, Carvalho CMRG, Paz SMRS. Avaliação do consumo alimentar de vitamina $A$ de crianças assistidas em creches comunitárias, Teresina (PI), Brasil. Nutrire. 2001; 22:7-19.

28. Marinho HA, Roncada MJ. Ingestão e hábitos alimentares de três capitais da Amazônia Ocidental brasileira: um enfoque especial à ingestão de vitamina A. Acta Amazônica. 2003; 33(2):263-74

29. Humphrey J, Friedman D, Natadisastra G, Muhilal. 24-hour history is more closely associated with vitamin A status and provides a better estimate of dietary vitamin A intake of deficient Indonesian preschool children than a food frequency method. J Am Diet Assoc. 2000; 100(12):1501-10.

30. Zohouri FV, Rugg-Gunn AJ. Sources of dietary iron in urban and provincial 4-year-old children in Iran. Asia Pacific J Clin Nutr. 2002; 11(2):128-32.

31. Cruz, ATR. O consumo alimentar de crianças: avaliação pelo "Método da Pesagem Direta" em 3 creches no Município de São Paulo - SP [dissertação]. São Paulo: Universidade de São Paulo; 2001.

32. Cadorna CML. Avaliação do consumo alimentar de crianças freqüentadoras de creches municipais de São Paulo [dissertação]. São Paulo: Universidade de São Paulo; 1999.

33. Goodwin RA, Buckholz AC, McKim MK, Stuart B, O'Connor DL. Caregiving arrangement and nutrition: good news with some reservations. Can J Pub Health. 1999; 90(1):45-51.

34. Fisberg RM, Slater B, Marchioni DL. Como estimar a probabilidade de adequação e inadequação da ingestão de nutrientes. Nutr Pauta. 2003; 58: 29-33.

35. Barr SI, Murphy SP, Poos MI. Interpreting an using dietary reference intakes in dietary assessment of individuals and groups. J Am Diet Assoc. 2002; 102(6):780-8.

36. Castro TG, Novaes JF, Silva MR, Costa NMB, Franceschini SCC, Tinoco ALA, et al. Caracterização do consumo alimentar, ambiente socioeconômico e estado nutricional de pré-escolares de creches municipais. Rev Nutr. 2005; 18(3):321-30.

37. Takyi EEK. Nutrition status and nutrient intake of preschool children in Northern Ghana. East African Med J. 1999; 76(9):510-5.

38. Wyatt CJ, Triana Tejas MA. Nutrient intake and growth of preschool children from different socioeconomic regions in the City of Oaxaca, México. Ann Nutr Metab. 2000; 44(1):14-20.

39. Dennison BA, Rockwell HL, Baker SL. Fruit and vegetable intake in young children. J Am Coll Nutr. 1998; 17(4):371-8.

40. Muñoz KA, Krebs-Smith SM, Ballard-Boubash R, Cleveland LE. Food intakes of US children and adolescents compared with recomendations. Pediatrics. 1997; 100(3):323-9.

41. Beaton GH, Burema J, Ritenbaugh C. Errors in the interpretation of dietary assessments. Am J Clin Nutr. 1997; 65(4 Suppl):1100S-7.

42. Scagliusi FB, Lancha Júnior AH. Subnotificação da ingestão energética na avaliação do consumo alimentar. Rev Nutr. 2003; 16(4):471-81.

43. Oliveira SP, Thébaud-Mony A. Estudo do consumo alimentar: em busca de uma abordagem 
multidisciplinar. Rev Saúde Pública.1997; 31(2):201-8.

44. Ortega RM, Requejo AM, Navia B, Lopez-Sobaler AM, Andres P, et al. Effect of saturated fatty acid consumption on energy and nutrient intake and blood lipid levels in preschool children. Ann Nutr Metab. 2001; 45(3):121-7.

45. Bollella MC, Boccia LA, Nicklas TA, Lefkowitz KB, Pittman BP, Zang EA, et al. Assessing dietary intake in preschool children. The healthy start project-New York. Nutr Res. 1999; 19(1):37-48.

46. Leone C. Avaliação da condição nutricional. In: Nóbrega, FJ. Distúrbios da nutrição. Rio de Janeiro: Revinter; 1998. p.65-70.

47. Yuyama LKO, Vasquez ALV, Aguiar JPL, Macedo SHM, Yokenura L, Nagahama D, et al. Composição química e adequação de uma instituição beneficiente de Manaus, Amazonas, Brasil. Acta Amazônica. 1999; 29(4):549-54.

48. Atkin LM, Davies PSW. Diet composition and body composition in preschool children. Am J Clin Nutr. 2000; 72(1):15-21.
49. Cruz GF, Santos RS, Carvalho CMRG, Motta GC. Avaliação dietética em creches municipais de Teresina, Piauí, Brasil. Rev Nutr. 2001; 14(1): 21-32.

50. Spinelli MG, Goulart RMM, Santos ALP, Gumiero LC, Farhud CC, Freitas EB, et al. Consumo alimentar de crianças de 6 a 18 meses em creches. Rev Nutr. 2003; 16(4):409-14.

51. Holland CV, Szarfac SC. Consumo energético do pré-escolar de creches. Nutrire. 2003; 25:61-70.

52. Levy-Costa RB, Monteiro CA. Consumo de leite de vaca e anemia na infância no município de São Paulo. Rev Saúde Pública. 2004; 38(6):797-803.

53. Bosscher D, Caillie-Bertrand MV, Deelstra H. Daily dietary fibre intake of children, 2 to 3 years of age, living in Antwerp, Belgium. Nutr Res. 2002; 22:1401-11. 
726 | R.C. FALCĀO-GOMES et al.

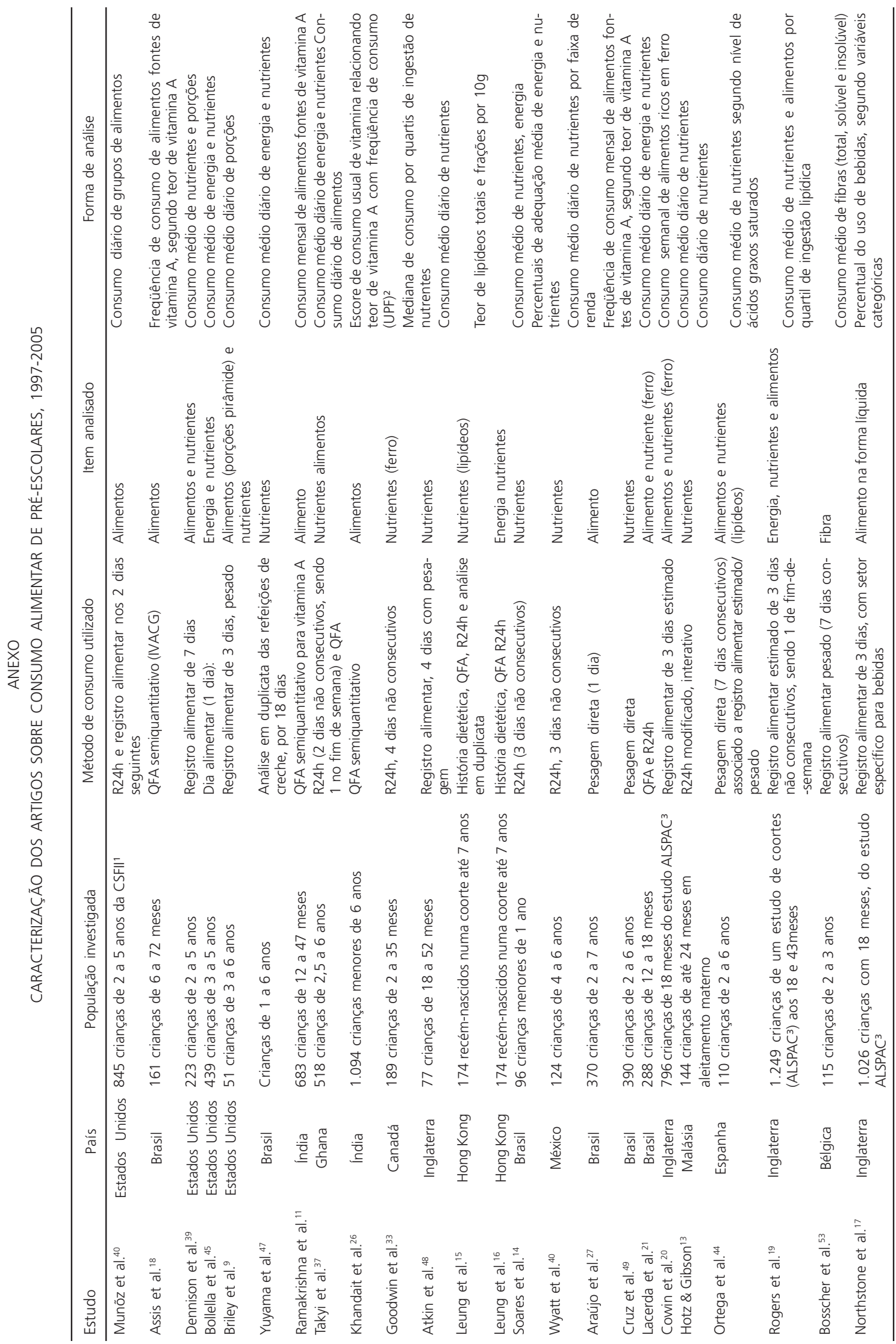


CONSUMO ALIMENTAR EM PRÉ-ESCOLARES | 727

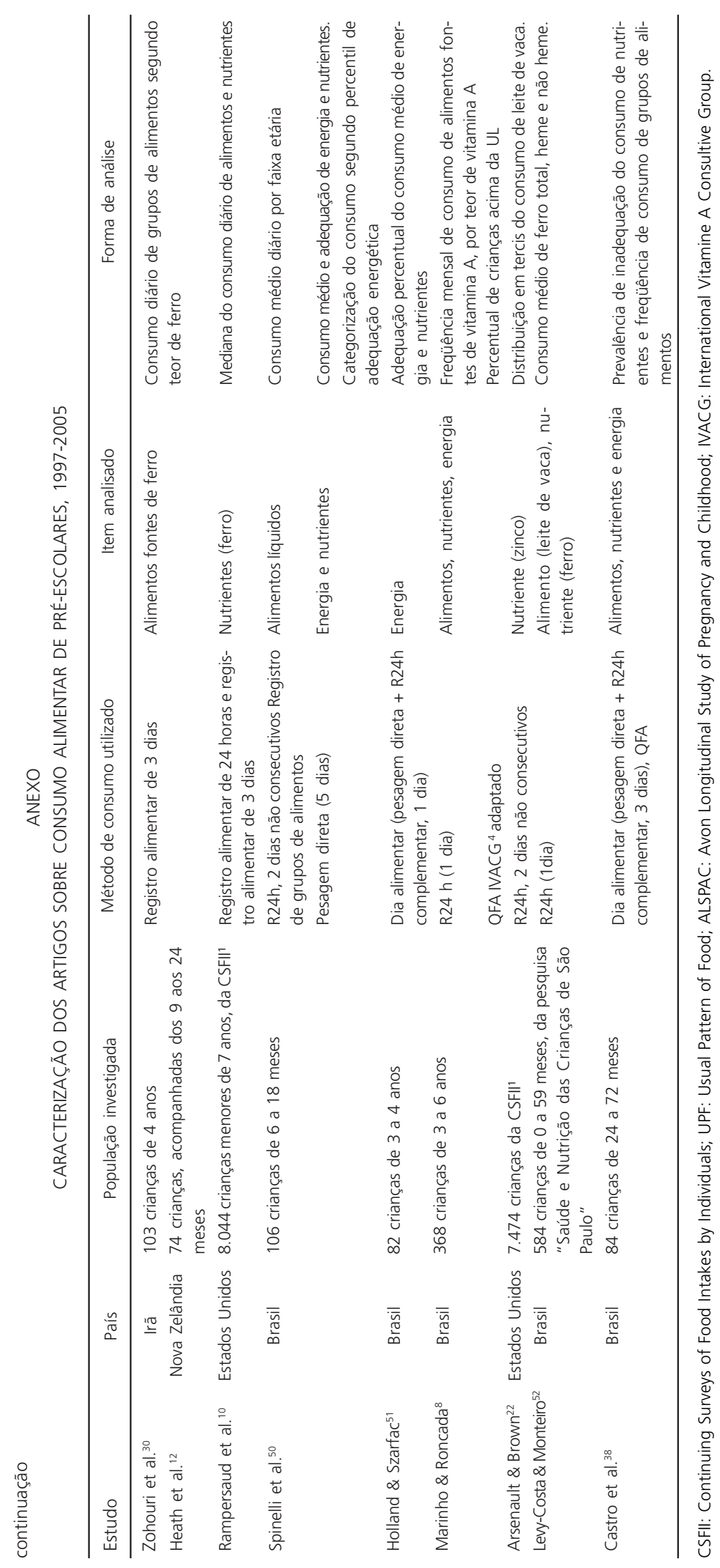


